\title{
DESARROLLO DE LA RESISTENCIA EN JUGADORAS DE FÚTBOL: MÉTODO INTERMITENTE Vs MÉTODO CONTINUO
}

Acosta P. ${ }^{1}$, Sanabria Y. ${ }^{1}$, Agudelo $C^{2}$.

1 Docente Universidad Pedagógica y Tecnológica de Colombia -UPTC E-mail acostajon14@hotmail.com paulo.acosta@uptc.edu.co.

1 Docente Universidad Pedagógica y Tecnológica de Colombia -UPTC Coordinador Maestría en Pedagogía de la Cultura Física E-mail yofre.sanabria@uptc.edu.co, yofresana6@gmail.com

2 Docente Universidad de Antioquia -UDEA Coordinador del programa en Entrenamiento Deportivo del Instituto Universitario de Educación Física, E-mail carlos.agudelo@udea.edu.co , agudelojudoka@gmail.com

\section{RESUMEN}

Este estudio tuvo como objetivo comparar los métodos de entrenamiento (continúo e intermitente) en la resistencia aeróbica de las jugadoras de fútbol de la Universidad Pedagógica y Tecnológica de Colombia (UPTC) seccional Chiquinquirá. Para esto 33 jugadoras fueron divididas aleatoriamente en tres grupos: grupo control, grupo experimental de entrenamiento con método continúo y grupo experimental de entrenamiento con método intermitente, La intervención se realizó durante 8 semanas, con 3 sesiones semanales de entrenamiento con el énfasis respectivo. Al comenzar y al finalizar los programas se les aplicó a los jugadores el Test de Léger para valorar la potencia aeróbica máxima determinando el $\mathrm{VO}_{2}$ Máx. Se puede señalar que los planes de entrenamiento generaron un efecto significativo en las deportistas ya que los análisis muestran que existen cambios significativos entre el pretest y el postest de cada uno de los grupos $(p>0,05)$. Los resultados de este estudio indican que 8 semanas de entrenamiento con métodos (continúo e intermitente) buscando desarrollar la resistencia en jugadores de fútbol sala son suficientes para conseguir adaptaciones positivas en esta capacidad física para jugadoras universitarias, siendo el método intermitente el que obtuvo mejoras superiores en la mejora del $\mathrm{VO}_{2}$ Máx.

Palabras clave: método continuo, método intermitente, resistencia aeróbica.

\section{DEVELOPMENT OF RESISTANCE IN SOCCER PLAYERS: CONTINUOUS METHOD Vs INTERMITTENT METHOD}

\begin{abstract}
ABSTRAC
This study was undertaken as a objective to compare the methods of training (continuous and intermittent) in the aerobic resistance of the players of soccer of the Pedagogic and Tecnologic of Colombia University (UPTC) sectional Chiquinquirá. To do this 33 players were divided randomly in three groups: group informed control, experimental group of training with method continued and experimental group of training with intermittent method, The intervention was realized for 8 weeks, with 3 weekly meetings training sessions with the respective emphasis. At the beginning and end of the programs, were applied to the players the Test of Léger to value the aerobic maximum potency determining the maximum $\mathrm{VO}_{2}$. Can be noted that training plans did generate
\end{abstract}


a significant effect in athletes since the analysis show that there are significant changes between the pretest and the posttest for each of the groups $(p>0,05)$. The results of this study indicate that 8 weeks of training methods (continuous and intermittent) looking about how to develop the resistance in players of soccer are sufficient to obtain positive adaptations in this physical capacity for university players, being the intermittent method obtained top progress in the progress of the VO2 Máx.

Keywords: method continued, intermittent method, aerobic resistance.

Training.

\section{INTRODUCCIÓN}

El fútbol es una disciplina deportiva ligada a cambios (físicos, psicológicos, metabólicos entre otros) durante el ambiente competitivo, el ciclo de sus acciones es relativo, y no se puede determinar de antemano las circunstancias que van a determinar el contexto del juego. Como lo afirma (Alvarez, 2000) la preparación física es fundamental, la resistencia es la capacidad que tiene el individuo para mantener el mayor tiempo posible un esfuerzo eficaz, con el máximo aprovechamiento de oxígeno requerido. (Forteza, 1994). (Arjol, 2.000), dice que en el caso del futbolista la cualidad de resistencia implica retrasar al máximo la aparición de la fatiga manteniendo un esfuerzo medio-alto a lo largo de encuentro, impidiendo que el cansancio altere la percepción, toma de decisión y ejecución de las distintas acciones que se producen a lo largo de un encuentro; y por lo tanto mantener un ritmo de juego alto.

El fútbol femenino ha florecido en los últimos años y despierta en la actualidad un interés sin precedentes. La FIFA cree que el fútbol femenino tiene todavía un mayor potencial de crecimiento y por ello promociona el fútbol femenino de manera activa en todo el mundo mediante nuestras competiciones y eventos, campañas de sensibilización y programas de desarrollo. (FIFA, 2016)

Para la realización de esta investigación, se utilizaron el método continúo que consiste en realizar un esfuerzo físico sin interrupción ni pausas; Los sistemas continuos se caracterizan porque las acciones son mantenidas durante un periodo largo de tiempo. La intensidad es constante y moderada, y el volumen bastante grande (Fortó). El ejercicio intermitente es el que alterna períodos de ejercicio intenso, intercalados con períodos de recuperación a baja intensidad 0 en reposo. (Daniels, J y Scardina, N., 1984).

Por todo ello, el objetivo de este estudio fue comparar los métodos de entrenamiento (continuo e intermitente) en la resistencia de las jugadoras de fútbol de la Universidad Pedagógica y Tecnológica de Colombia (UPTC) seccional Chiquinquirá.

\section{MATERIAL Y MÉTODOS}

\section{Sujetos}

Treinta y tres jóvenes universitarias jugadoras de fútbol (19.3 \pm 1.23 años) $(160.3 \pm 5.42 \mathrm{~cm})(56.8 \pm 8.66 \mathrm{Kg})$ pertenecientes a la UPTC seccional Chiquinquirá hicieron parte del estudio. Todas las jugadoras tenían experiencia previa en entrenamiento $(2.818 \pm 1.975$ años), no reportaron limitaciones físicas 0 lesiones musculo-esqueléticas que pudieran afectar el proceso de entrenamiento $y$ testaje, todo soportado por un examen médico realizado previamente al inicio del proceso. Todas las participantes fueron informadas en detalle acerca de los procedimientos experimentales y los posibles riesgos y beneficios de esta investigación. El estudio obtuvo el consentimiento informado por escrito de 
todos los atletas antes de comenzar la fase experimental.

\section{Procedimiento}

A las jugadoras se les realizó un pre-test (test de Léger), luego se dividieron aleatoriamente en grupo control, grupo experimental e entrenamiento con método continuo y grupo experimental de entrenamiento con método intermitente.

El grupo control continuo con sus tareas cotidianas de entrenamiento, al grupo de entrenamiento con método continuo se les aplicó una tarea que consistía en realizar carrera continua durante 20 minutos con balón, La distancia que se debia recorrer osciló entre $3.4 \mathrm{~km}$ y $4.4 \mathrm{~km}$, de acuerdo a los datos arrojados por el pretest para cada grupo de jugadoras y esta dentro de el rango de acuerdo a los parametros arrojados por la (FIFA., 2011) donde el promedio de recorrido corresponde a una distancia en posesión del balón (entre 3.200 $\mathrm{m}$ y $3.450 \mathrm{~m}$ ), esta tarea la realizaron en cada sesión. (Estructura de trabajo 1)

El grupo de entrenamiento con método intermitente, realizó en cada una de las sesiones 3 tareas, con una relación 1-1 donde el tiempo de trabajo era de 20 segundos, y que consistían en 1) realizar un ejercicio de velocidad de desplazamiento, la distancia que debían recorrer las jugadoras variaba de acuerdo a los resultados obtenidos en el pretest. 2) ejercicio de coordinación, El ejercicio consistió en realizar tres golpes dentro de un aro con los pies alternándolos (derecha- izquierdaderecha) y pasar al siguiente aro y repetir el movimiento esta vez (izquierda-derechaizquierda), continuando la secuencia hasta finalizar el tiempo, se realizaba lo más rápido posible. 3) ejercicio de fundamentación técnica: consistió en conducir el balón en forma de zig- zag por entre los conos lo más rápido posible, sin hacer contacto con los mismos. (Estructura de trabajo 2)

Al finalizar las 8 semanas de intervención se procedió a realizar el pos-test y una vez terminado, se realizó el análisis e interpretación de resultados de pretest y pos-test, para observar si los programas aplicados contribuyeron al mejoramiento del $\mathrm{VO}_{2}$ Máx. en las jugadoras de fútbol de la UPTC seccional Chiquinquirá.

\section{Resultados}

Los datos fueron analizados utilizando el programa estadístico SPSS v.13 estudiantil, inicialmente se hizo un análisis exploratorio de datos mediante estadística descriptiva, luego se plantearon y probaron las hipótesis asociadas con el porcentaje de mejora relacionados con los métodos continuo e intermitente en los grupos de estudio y en el grupo control, a través de procedimientos inferenciales.

En la Tabla 1 se presentan los datos del $\mathrm{VO}_{2}$ Máx. de las deportistas involucradas en el estudio y los porcentajes de mejora a nivel individual y de forma grupal incluyendo los datos del pretest, del postest y del porcentaje individual de mejora.

Referente al grupo control se observa que 6 jugadoras mantuvieron estables sus valores del $\mathrm{VO}_{2}$ Máx., es decir, obtuvieron los mismos valores tanto en el pretest como en el postest sin lograr ningún porcentaje individual de mejora; las 5 restantes mejoraron su $\mathrm{VO}_{2}$ Máx. en el postest obteniendo porcentajes individuales de $9.2 \%$ en tres casos y de $8.43 \%$ en dos casos; Los anteriores resultados generaron un porcentaje de mejora grupal de un $3.97 \%$ el cual comparado con los otros dos grupos (método continuo e intermitentes) es el más bajo.

En lo que respecta al grupo entrenado con el método continuo, se observa que 4 jugadoras mantuvieron estables sus valores 
del $\mathrm{VO}_{2}$ Máx., es decir, obtuvieron los mismos valores tanto en el pretest como en el postest sin lograr ningún porcentaje individual de mejora; las 7 restantes mejoraron su $\mathrm{VO}_{2}$ Máx. en el postest obteniendo porcentajes individuales de $10.1 \%$ en un caso, de $8.4 \%$ en dos casos, de $16.9 \%$ en otro caso, de $18.4 \%$ en el mejor de los casos, de $9.2 \%$ y $7.2 \%$ en los dos casos restantes; Los anteriores resultados generaron un porcentaje de mejora.
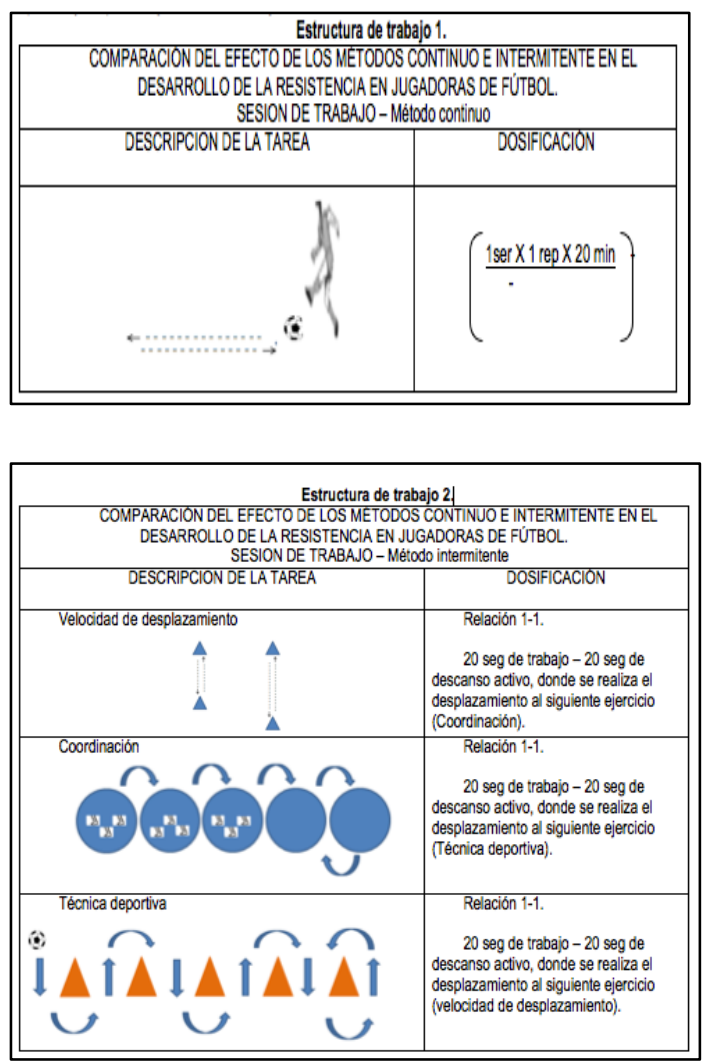

\begin{tabular}{|c|c|c|c|c|c|c|c|c|c|}
\hline \multicolumn{10}{|c|}{$\mathrm{VO}_{2}$ Máximo (m/ $\left./ \mathrm{kg} / \min \right)$} \\
\hline \multirow[t]{2}{*}{ SUJETO } & \multicolumn{3}{|c|}{ GRUPO CONTROL } & \multicolumn{3}{|c|}{ GRUPO CONTINUO } & \multicolumn{3}{|c|}{ GRUPO INTERMITENTE } \\
\hline & Pretest & Postets & $\begin{array}{l}\text { \% Individual } \\
\text { de mejora }\end{array}$ & Pretest & Postets & $\begin{array}{l}\% \text { Individual } \\
\text { de mejora }\end{array}$ & Pretest & Postets & $\begin{array}{l}\text { \% Individual } \\
\text { de mejora }\end{array}$ \\
\hline 1 & 32.6 & 32.6 & 0 & 29.6 & 32.6 & 10.14 & 29.6 & 35.6 & 20.27 \\
\hline 2 & 32.6 & 32.6 & 0 & 35.6 & 35.6 & 0.00 & 29.6 & 35.6 & 20.27 \\
\hline 3 & 32.6 & 32.6 & 0 & 35.6 & 38.6 & 8.43 & 32.6 & 38.6 & 18.40 \\
\hline 4 & 32.6 & 35.6 & 9.20 & 35.6 & 41.6 & 16.85 & 35.6 & 38.6 & 8.43 \\
\hline 5 & 32.6 & 35.6 & 9.20 & 32.6 & 38.6 & 18.40 & 38.6 & 44.6 & 15.54 \\
\hline 6 & 32.6 & 35.6 & 9.20 & 32.6 & 35.6 & 9.20 & 38.6 & 41.6 & 7.77 \\
\hline 7 & 35.6 & 35.6 & 0 & 35.6 & 38.6 & 8.43 & 38.6 & 41.6 & 7.77 \\
\hline 8 & 35.6 & 38.6 & 8.43 & 41.6 & 44.6 & 7.21 & 38.6 & 44.6 & 15.54 \\
\hline 9 & 35.6 & 38.6 & 8.43 & 38.6 & 38.6 & 0.00 & 38.6 & 44.6 & 15.54 \\
\hline 10 & 38.6 & 38.6 & 0 & 38.6 & 38.6 & 0.00 & 41.6 & 44.6 & 7.21 \\
\hline 11 & 38.6 & 38.6 & 0 & 38.6 & 38.6 & 0.00 & 41.6 & 47.6 & 14.42 \\
\hline Promedios & 34.50 & 35.87 & & 35.87 & 38.32 & & 36.69 & 41.6 & \\
\hline $\begin{array}{c}\% \\
\text { de mejora } \\
\text { grupal }\end{array}$ & & 3.97 & & & 6.83 & & & 13.38 & \\
\hline
\end{tabular}

Tabla 1.Resultados $\mathrm{VO}_{2}$ Máx. y porcentajes de mejora a nivel individual y grupal. grupal de un $6.83 \%$ el cual es más alto que el obtenido por las jugadoras del grupo control.

En cuanto al grupo entrenado con el método intermitente, en la Tabla 1 se observa que las 11 jugadoras mejoraron su $\mathrm{VO}_{2}$ Máx. en el postest obteniendo porcentajes individuales de $20.271 \%$ en dos casos siendo los más altos de este grupo, de $18.40 \%$ en otro caso, de $8.43 \%$ en otra jugadora, de $15.54 \%$ en tres casos, de $7.77 \%$ en dos jugadoras, $7.21 \%$ en otro caso y de $14.42 \%$ en el último caso. Los anteriores resultados generaron un porcentaje de mejora grupal de un $13.38 \%$ el cual es más alto que el obtenido por las jugadoras del grupo control y del grupo de jugadoras entrenadas con el método continuo.

Se realizó la prueba t-student a cada grupo (tabla 2) con un nivel de significancia $a=$ 0.05. En la Tabla 2 se observa que el Pvalor de la prueba (Sig. 2-tailed) para el grupo control es de 0.016, para el grupo entrenado con el método continuo es de 0.005 y para el grupo entrenado con el método intermitente es de 0.000 , los cuales son inferiores al 0.05 lo cual indica que las diferencias en el $\mathrm{VO}_{2}$ Máx. si son significativas en promedio.

En síntesis, los resultados permiten establecer que hay un porcentaje de mejora significativo en el $\mathrm{VO}_{2}$ Máx. al comparar los valores en el pretest con los del postest en el grupo control, en el grupo entrenado con el método continuo y en el grupo entrenado con el método intermitente; sin embargo, el porcentaje de mejora grupal es mayor en el grupo entrenado con el método intermitente en relación con los otros dos grupos. 


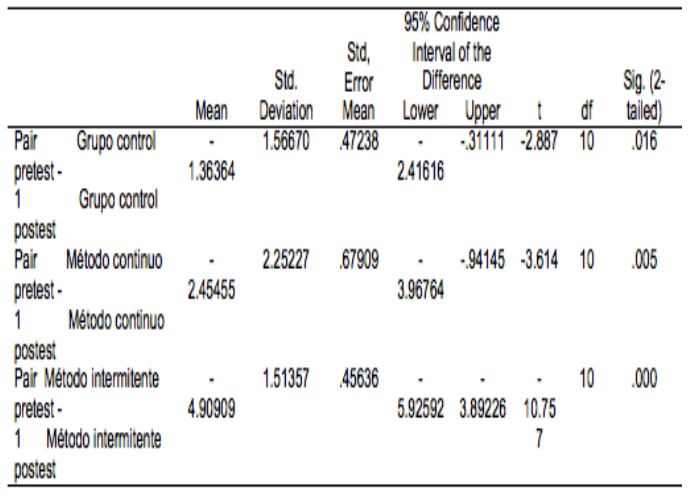

Tabla 2 Prueba t-student para los datos de los grupos, Paired Diferences.

En la tabla 3 se deduce que los porcentajes de mejora de las jugadoras entrenadas con el método intermitente son mayores que sus correspondientes para las jugadoras del grupo continuo excepto en cuatro casos donde resultaron iguales o inferiores, y para las jugadoras del grupo control excepto en dos casos donde resultaron iguales 0 inferiores, el promedio 4.04 corresponde al porcentaje de mejora grupal de $3.97 \%$ en el grupo control, el promedio de 7.15 corresponde al porcentaje de mejora grupal de $6.83 \%$ en el grupo continuo y el promedio de 13.74 corresponde al porcentaje de mejora grupal de $13.38 \%$ en el grupo intermitente; estos resultados proporcionan indicios de que el método intermitente es mejor que el método de entrenamiento usado con el grupo control y que el método de entrenamiento utilizado con el grupo continuo.

Se aplicó las pruebas t-student para muestras independientes relacionando los grupos, tabla 4, además se consideró tanto la homocedasticidad (varianzas iguales) como la heterocedasticidad (varianzas distintas), y se pudo concluir que el método continuo no difiere significativamente del método de entrenamiento aplicado al grupo control.

También en la tabla 4, se puede concluir que el método intermitente difiere significativamente del método de entrenamiento aplicado a los grupos continuo y control, y genera unos porcentajes de mejora grupal significativamente mayores que estos.

\begin{tabular}{cccc} 
Deportista & $\begin{array}{c}\text { Porcentaje } \\
\text { individual de } \\
\text { mejora grupo } \\
\text { control }\end{array}$ & $\begin{array}{c}\text { Porcentaje } \\
\text { individual de } \\
\text { mejora grupo } \\
\text { continuo }\end{array}$ & $\begin{array}{c}\text { Porcentaje } \\
\text { individual de } \\
\text { mejora grupo } \\
\text { intermitente }\end{array}$ \\
\hline 1 & 0.00 & 10.14 & 20.27 \\
2 & 0.00 & 0.00 & 20.27 \\
3 & 0.00 & 8.43 & 18.40 \\
5 & 9.20 & 16.85 & 8.43 \\
6 & 9.20 & 18.40 & 15.54 \\
7 & 9.20 & 9.20 & 7.77 \\
8 & 0.00 & 8.43 & 7.77 \\
9 & 8.43 & 7.21 & 15.54 \\
10 & 8.43 & 0.00 & 15.54 \\
11 & 0.00 & 0.00 & 7.21 \\
\hline Promedio & 0.00 & 0.00 & 14.42 \\
\hline Tabla 3 Porcentaje de mejora & 7,15 & 13,74 \\
\hline
\end{tabular}

En síntesis, el método intermitente es mejor que el método continuo y que el método de entrenamiento aplicado al grupo control. Lo anterior permite afirmar que el porcentaje de mejora grupal del $\mathrm{VO}_{2}$ Máx. correspondiente a las jugadoras entrenadas con el método intermitente es significativamente mayor que el porcentaje de mejora para las jugadoras entrenadas con el método continuo o las del grupo control.

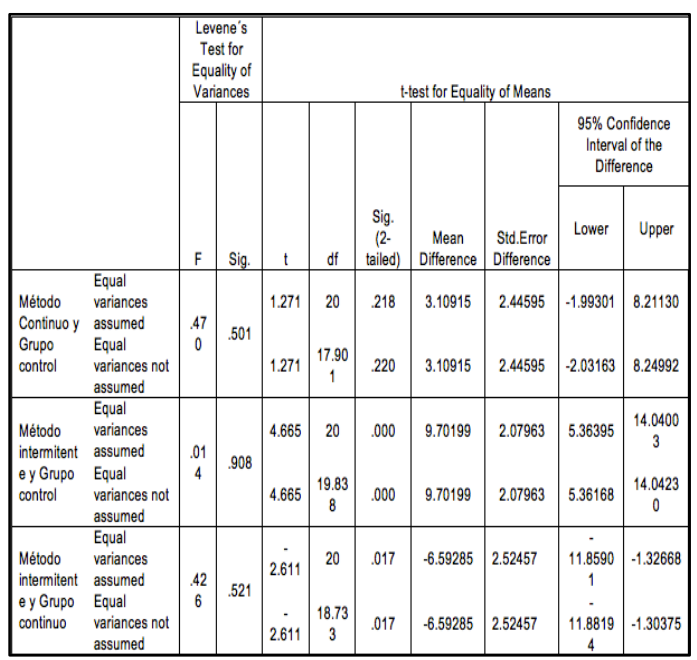

Tabla 4 Prueba t-student para muestras independientes

\section{Discusión}

El propósito del presente estudio fue comparar el efecto de los métodos continuo e intermitente en el desarrollo de la 
resistencia aeróbica en jugadoras de fútbol, hallando el $\mathrm{VO}_{2}$ Máx. mediante el test de Léger, esto ya que en la actualidad la gran mayoría de los deportes busca obtener el rendimiento más apropiado de los deportistas a través del entrenamiento deportivo y donde los planes de entrenamiento se deben dirigir hacia la obtención de ese fin, para este caso la resistencia.

Según la variable planteada, del $\mathrm{VO}_{2}$ Máx., se reconoce que hubo cambios de mejora significativa después de la aplicación del entrenamiento de resistencia a partir de los métodos continuo e intermitente.

Los tramos intensos de 20 minutos para el método continuo se encuentran entre las cuantificaciones recomendadas para el entrenamiento de la potencia aeróbica en los deportes de conjunto (Fortó, J., s.f), y en relación al método intermitente están acordes a los aportados por (Rivas, M. \& Sánchez, E., 2013) que manifiestan que en el entrenamiento fraccionado se recomienda trabajar entre 20 a 25 min y donde afirman que para el fútbol se consideran como más pertinentes las siguientes variantes: 15-15, $20-20,10-20$ y $15-30$ para la mejora del $\mathrm{VO}_{2}$ Máx.

Las intensidades con las que se trabajó en el presente estudio, y con el objetivo de mejorar el $\mathrm{VO}_{2}$ Máx. siguiendo los lineamientos de (López, J. \& Fernández, A., 2006) estuvieron entre $70 \%-83 \%$. Pudiéndose afirmar que se trabajó con intensidades similares a las de competencia, teniendo como parámetro de intensidad el porcentaje del $\mathrm{VO}_{2}$ Máx. que se encuentra entre $65 \%-95 \%$ del $\mathrm{VO}_{2}$ Máx. (Palavecino, 2002) y $75 \%$ del $\mathrm{VO}_{2}$ Máx. (Bangsbo, 1994).

Los resultados obtenidos en el presente estudio están en concordancia con lo expuesto por (Rivas, M. \& Sánchez, E., 2013) donde indican que el aumento de la potencia aeróbica producido por la carrera intermitente, es más eficiente que el producido por la carrera continua, ya que la carrera intermitente tiene mayor similitud a los esfuerzos intermitentes que se producen en un partido. Coincidiendo con el estudio realizado por (Arecheta, C. Gómez, M. \& Lucia, A., 2006) estos resultados demuestran que un alto $\mathrm{VO}_{2}$ Máx. ayuda a realizar y mantener esfuerzos máximos en los tiempos finales de un partido, donde la fatiga de las futbolistas es más elevada, $y$ con el estudio realizado por (Kohan, A. Figueroa, R. \& Dolce, P., 2003) que afirma que las necesidades del fútbol moderno desde el aspecto de la preparación física se considera importante realizar patrones de ejercicio que nos permitan estimular la potencia aeróbica con relaciones trabajo pausa similares a las producidas en competencia

Al comparar el estudio de (Ramírez, J. Muros, J. Morente, J. Sánchez, C. Femia, P.\& Zabala, M., 2012) donde se determinó el efecto de un programa de entrenamiento aeróbico de alta intensidad de 8 semanas, desarrollado durante las clases de educación física, sobre la capacidad aeróbica de adolescentes de 15 a 18 años, donde contó con un total de 84 adolescentes (51 chicos y 33 chicas). El estudio concluyó que un programa de entrenamiento aeróbico de alta intensidad de 8 semanas, 2 días por semanas, mejora la capacidad aeróbica de los alumnos. Una sesión extra de ejercicio intenso por semana supone una mayor mejora en las chicas, no produciendo tales efectos en los chicos; resultados que soportan los obtenidos en el presente estudio donde independiente del método de entrenamiento y 10 grupo se obtiene mejoras en el $\mathrm{VO}_{2}$ Máx.con 8 semanas de entrenamiento.

(Muñoz, 2008) realizó un estudio sobre la influencia del método continuo invariable en el desarrollo de la resistencia aeróbica, en los jugadores del semillero de fútbol de la Universidad de Antioquia, con edades entre los 17 y 24 años, y concluyó que un plan de entrenamiento de 20 sesiones, influyó en el 
desarrollo de la resistencia aeróbica en los jugadores. Los resultados mostraron que los jugadores incrementaron el $\mathrm{VO}_{2}$ Máx. (de $50,45$ a $54,9 \mathrm{ml} / \mathrm{kg} / \mathrm{min}:<0,05)$, para efectos del presente estudio los resultados obtenidos por parte del grupo de entrenamiento con método continuo (de $35,87$ a $38,327 \quad \mathrm{ml} / \mathrm{kg} / \mathrm{min}: \quad<0.05)$ demuestran que con 24 sesiones se pueden obtener mejoras significativas en el $\mathrm{VO}_{2}$ Máx., y afirmando lo dicho por (Morante, 1994) que en el fútbol se puede mejorar la resistencia aeróbica utilizando la carrera continua 0 mediante un ejercicio de conducción, pases por parejas, etc.

Con relación a las mejoras del $\mathrm{VO}_{2}$ Máx., se puede decir que las jugadoras que presentaron mejores resultados fueron las que más bajo nivel obtuvieron en el pretest (en el grupo de entrenamiento con método intermitente), y esto puede obedecer a que las adaptaciones ocurren según el nivel inicial de entrenamiento del sujeto. Se está de acuerdo con la afirmación de (Pardo, 2006) que dice que, en sujetos con un nivel de rendimiento alto, las adaptaciones serán relativamente menos significativas, y con la suposición expuesta por (Guzman, J. Jimenez, J., 2013) acerca de que las planificaciones, más allá de categorizarlas por edades, deben ser un trabajo más desde la individualidad.

Además y comparando los resultados aportados por (Barbero, J. y Barbero, V., 2007) del incrementó del máximo consumo de oxígeno tras siete semanas de entrenamiento el grupo mejoró en un $6.45 \%$ $\pm 9.11 \mathrm{ml} / \mathrm{kg} / \mathrm{min}$, para el presente estudio el grupo control mejoró $3.97 \% \pm 4.65$ $\mathrm{ml} / \mathrm{kg} / \mathrm{min}$, el grupo con método continuo obtuvo una mejora de $6.83 \% \pm 6.64$ $\mathrm{ml} / \mathrm{kg} / \mathrm{min}$ y el grupo con método intermitente aumento su $\mathrm{VO}_{2}$ Máx. en un $13.38 \% \pm 5.09 \mathrm{ml} / \mathrm{kg} / \mathrm{min}$ en ocho semanas de intervención, por lo que se puede afirmar que en un periodo de ocho semanas se obtienen mejoras en esta capacidad, confirmando lo dicho por (Kohan, 2003) para producir mejoras en el consumo de oxígeno se necesita un tiempo mínimo de estimulación sistemática que oscila entre 6 y 8 semanas .

Los resultados finales del $\mathrm{VO}_{2}$ Máx. obtenidos por las jugadoras en este estudio son similares (grupo control $35.87 \pm 2.49$ $\mathrm{ml} / \mathrm{kg} / \mathrm{min}^{-1} ;$ grupo de entrenamiento continuo $38.327 \pm 3.13 \mathrm{ml} / \mathrm{kg} / \mathrm{min}^{-1}$ y el grupo de entrenamiento intermitente 41.60 $\pm 4.024 \mathrm{ml} / \mathrm{kg} / \mathrm{min}^{-1}$ ) a los estudios realizados con población de género femenino, como por ejemplo el adelantado por (Miles, A. Maclaren, D. Reilly, T. \& Yamanaka, K., 1993) con valores de (42.5 $\left.\mathrm{ml} / \mathrm{kg} / \mathrm{min}^{-1}\right)$ con 10 jugadoras inexpertas, el de (Reiter, L. Prouten, L. Rigney, L. Lambert, S. Estell, J. \& Barnsley, L., 1996) con valores de $\left(42.4 \pm 6.1 \mathrm{ml} / \mathrm{kg} / \mathrm{min}^{-1}\right)$ con $n=11$ jugadoras de primera división, un estudio adicional es el de (Tamer, K. Günay, M. Tiryaki, G. Cicioolu, I. \& Erol, E., 1997) con valores de $\left(43.15 \pm 4.06 \mathrm{ml} / \mathrm{kg} / \mathrm{min}^{-1}\right)$ con 22 jugadoras elite de Turquía, (Hoare, D. \& Warr, C., 2000) y su estudio arrojaron datos con valores de $\left(39.4 \pm 4.3 \mathrm{ml} / \mathrm{kg} / \mathrm{min}^{-}\right.$ 1) con $n=17$ jugadoras talentos de Australia, otro estudio es el de (Todd, M. Scott, D. \& Chisnall, P., 2002) con valores de (44.4 \pm $5.8 \mathrm{ml} / \mathrm{kg} / \mathrm{min}^{-1}$ ) que contó con una muestra de 120 jugadoras de Inglaterra y el estudio de (Polman, R. Walsh, D. Bloomfield, J. \& Nesti, M., 2004)con valores de (39.4 \pm 3.72 $\mathrm{ml} / \mathrm{kg} / \mathrm{min}^{-1}$ ) con 12 jugadoras de la primera división inglesa.

\section{REFERENCIAS BIBLIOGRÁFICAS}

Forteza. (1994). Entrenar para ganar.

Arjol, J. (2.000). entrenamiento de la resistencia en el fútbol. Retrieved Julio de 2015 from

www.fútbolpreparadoresfisicos.com

FIFA. (2016). fútbol femenino. From http://www.fifa.com

Fortó, J. ENTRENAMIENTO DE LA RESISTENCIA EN LOS DEPORTES COLECTIVOS. 
Daniels, J y Scardina, N. (1984). Interval training and performance. Sports Medicine , 1:327-34.

FIFA. (2011). Análisis del rendimiento físico durante la Copa Mundial Femenina de la FIFA Alemania 2011. Zurich.

Fortó, J. (s.f). Entrenamiento de la resistencia en deportes colectivos. Barcelona. España.

Rivas, M. \& Sánchez, E. (2013). Fútbol. Entrenamientoactual de la condición física del futbolista. In M. Salud. Heredia, Costa Rica.

López, J. \& Fernández, A. (2006). Fisiología del ejercicio ( $3^{\mathrm{a}}$ ed.). Argentina: Médica Panamericana.

Palavecino, N. (2002). Nutrición para el alto rendimiento. . España.

Bangsbo, J. (1994). Energy demands in competition soccer. . Journal of Sports Sciences , 5- 12.

Arecheta, C. Gómez, M. \& Lucia, A. (2006). La importancia del Vo2 máx.para realizar esfuerzos intermitentes de alta intensidad en el fútbol femenino de elite. Kronos, V, 412.

Kohan, A. Figueroa, R. \& Dolce, P. (2003). Comparación de variables fisiológicas y rendimiento alcanzado entre un ejercicio continuo vs. un ejercicio intermitente en futbolista amateur.

Ramírez, J. Muros, J. Morente, J. Sánchez, C. Femia, P.\& Zabala, M. (2012). Efecto de un programa de entrenamiento aeróbico de 8 semanas durante las clases de educación física en adolescentes. Nutrición hospitalaria , 747 - 754.

Muñoz, H. (2008). Influencia del método continuo invariable en el desarrollo de la resistencia aeróbica, en los jugadores del semillero de fútbol de la Universidad de
Antioquia con edades entre los 17 y 24 años de edad. Universidad de Antioquia - Instituto Universitario de Educación Física, Antioquia.

Morante, J. (1994). La Técnica como medio en el proceso de entrenamiento. Entrenamiento Deportivo , 25-27.

Pardo, J. (2006). Entrenamiento de la resistencia aeróbica. En: López,J.,

Fernández, A. Fisiología del ejercicio (Tercera ed.). Argentina: Médica Panamericana.

Guzman, J. Jimenez, J. (2013). Efectos de un plan de entrenamiento de resistencia sobre el VO2 máximo, la frecuencia cardíaca de reposo y los índices de recuperación en futbolistas juveniles. Viref , $2(4), 67$.

Barbero, J. y Barbero, V. (Marzo de 2007). Efectos del entrenamiento durante una pretemporada en la potencia máxima aerobica media mediante dos test de campo progresivos, uno continuo y uno intermitente. Melilla, Madrid, España.

Kohan, A. (2003). Entrenamiento del Metabolismo Aeróbico.

Miles, A. Maclaren, D. Reilly, T. \&

Yamanaka, K. (1993). An analysis of physiological strain in four-a-side women's soccer. In Reilly T., Clarys J. \& Stibbe A. Science and football II , 140-145.

Reiter, L. Prouten, L. Rigney, L. Lambert, S. Estell, J. \& Barnsley, L. (1996).

Physiological characteristics of female soccer players: laboratory and match-player assessments. (pp. 424 -425). Australian Conference of Science and Medicine in Sport.

Tamer, K. Günay, M. Tiryaki, G. Cicioolu, I. \& Erol, E. (1997). Physiological 
characteristics of Turkish Female Soccer players. Science and Footbal III , 37-39.

Hoare, D. \& Warr, C. (2000). Talent identification and women's soccer: an

Australian experience. J. Sports Sci , 751 758.

Todd, M. Scott, D. \& Chisnall, P. (2002).

Fitness characteristics of English female soccer players: an analysis by position and playing standard . In Spinks, W., Reilly, T. \&

Murphy, A. Science and Football IV , 374 381.

Polman, R. Walsh, D. Bloomfield, J. \& Nesti, M. (2004). Effective conditioning of female soccer players. J. Sports Sci , 191 -203. 